\title{
EUV reticle inspection using phase retrieval algorithms: a performance comparison
}

Ricarda Nebling, lacopo Mochi, Dimitrios Kazazis, Uldis Locans, Atoosa Dejkameh, et al.

Ricarda Nebling, lacopo Mochi, Dimitrios Kazazis, Uldis Locans, Atoosa Dejkameh, Yasin Ekinci, "EUV reticle inspection using phase retrieval algorithms: a performance comparison," Proc. SPIE 11147, International Conference on Extreme Ultraviolet Lithography 2019, 111470R (29 September 2019); doi: 10.1117/12.2536936

SPIE Event: SPIE Photomask Technology + EUV Lithography, 2019, Monterey, California, United States 


\title{
EUV reticle inspection using phase retrieval algorithms - a performance comparison
}

\author{
Ricarda Nebling, Iacopo Mochi, Dimitrios Kazazis, Uldis Locans, Atoosa Dejkameh, and Yasin \\ Ekinci
}

Paul Scherrer Institut, 5232 Villigen PSI, Switzerland

\begin{abstract}
RESCAN is an actinic patterned EUV mask metrology tool based on coherent diffraction imaging. An image of the reticle is reconstructed from recorded diffraction patterns using a phase retrieval algorithm. As semiconductor manufacturing has moved to EUV lithography to meet the next technology node, accurate photomask metrology with resolution in the nanometer range is crucial for high production yield. To find the optimal reconstruction strategy to achieve the highest resolution, sensitivity and reconstruction speed in RESCAN, we compared three algorithms. We demonstrate that, for the current setup, the best approach is the difference map algorithm.
\end{abstract}

Keywords: EUV mask inspection, APMI, CDI, lensless imaging, ptychography, phase retrieval algorithm

\section{INTRODUCTION}

RESCAN (REflective-mode EUV mask SCANning microscope) is a prototype for Extreme Ultra Violet (EUV) mask metrology developed at the Paul Scherrer Institut. ${ }^{1}$ It is based on Coherent Diffraction Imaging (CDI), a technique also referred to as lensless imaging. ${ }^{2,3}$ EUV photomasks are a key component of the lithography process for the manufacturing of semiconductor devices and they require accurate metrology at the nanometer scale to prevent eventual defects from reducing the production yield. A schematic of the RESCAN optical layout is shown in Fig. 1. The coherent EUV illumation from the synchrotron source is focused by a toroidal, multilayer coated mirror (M1) and folded onto the reticle by mirror M2 with a $6^{\circ}$ angle of incidence. From the reticle, the EUV illumination is reflected and propagated onto the detector where the diffraction pattern of the mask is recorded. As only the intensity (information on the diffraction pattern magnitude) is recorded but all phase information is lost, we need phase retrieval algorithms to reconstruct the (complex) image of the EUV reticle.

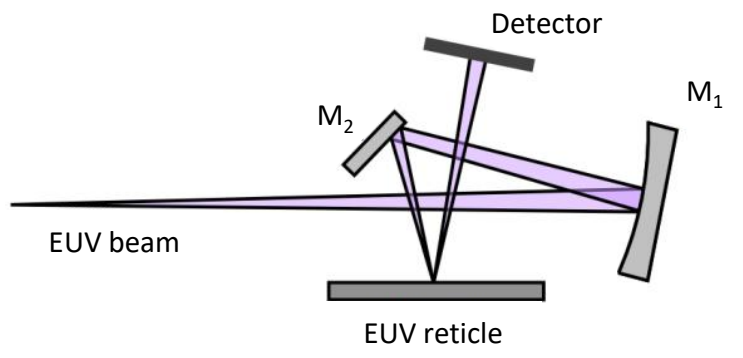

Figure 1. Schematic of the RESCAN setup.

Using CDI for actinic EUV reticle imaging has the advantage that no expensive and complex optical EUV optics are required. Furthermore, the magnitude and phase of the sample are reconstructed, allowing for amplitude defect detection alongside with the detection of phase defects. RESCAN can detect defects in die-to-die and

Further author information:

E-mail: ricarda.nebling@psi.ch

International Conference on Extreme Ultraviolet Lithography 2019, edited by Toshiro Itani,

Paolo A. Gargini, Patrick P. Naulleau, Kurt G. Ronse, Proc. of SPIE Vol. 11147, 111470R

(C) 2019 SPIE · CCC code: 0277-786X/19/\$21 · doi: 10.1117/12.2536936

Proc. of SPIE Vol. 11147 111470R-1

Downloaded From: https://www.spiedigitallibrary.org/conference-proceedings-of-spie on 13 Oct 2019 Terms of Use: https://www.spiedigitallibrary.org/terms-of-use 
die-to-database mode, where the reconstructed object is compared to a reference and the defects are detected as a difference in signal. ${ }^{1}$ We have demonstrated down to $50 \times 50 \mathrm{~nm}^{2}$ defect detection on mask for both, absorber and phase defects. ${ }^{4}$

As several phase retrieval algorithms have different performance characteristics, it is important to find the optimal strategy for mask inspection using RESCAN towards higher resolution, sensitivity and optimal reconstruction speed.

\section{PHASE RETRIEVAL ALGORITHMS}

RESCAN uses a CDI technique called ptychography to get a (complex) image of the EUV reticle. Historically, ptychography was proposed in the 1960's by Hegerl and Hoppe ${ }^{5}$ as a solution to the phase problem in scanning transmission electron microscopes. In short, a finite-sized coherent illumination probe scans the object (here the reticle) in partially overlapping positions. For each probe position, one corresponding diffraction pattern is recorded. The real space overlap of the probes introduces redundancy in the diffraction patterns, as two patterns from partially overlapping positions contain information from the same area of the sample. This redundancy allows for a high quality object reconstruction as well as a large field of view. ${ }^{6,7}$

Most phase retrieval algorithms used in ptychography are iterative and follow the general principle outlined in Fig. 2. From an initial (complex) object and probe guess, the exit wave $\Psi_{j}=O(\mathbf{r}) P\left(\mathbf{r}-\mathbf{r}_{j}\right)$ (the outgoing beam after being reflected by the reticle) is obtained. ${ }^{7}$ The exit wave is then propagated onto the detector plane using the Fourier transform (we consider here a detector in far-field), where it forms the complex amplitude diffraction pattern. The magnitude of the diffraction pattern is replaced by the magnitude measured on the detector. The new magnitude is recombined with the unchanged phase. Then the updated complex diffraction pattern is back-propagated to the object plane using the inverse Fourier transform, forming a new exit wave estimate from which the object guess is updated. This is repeated until convergence is reached.

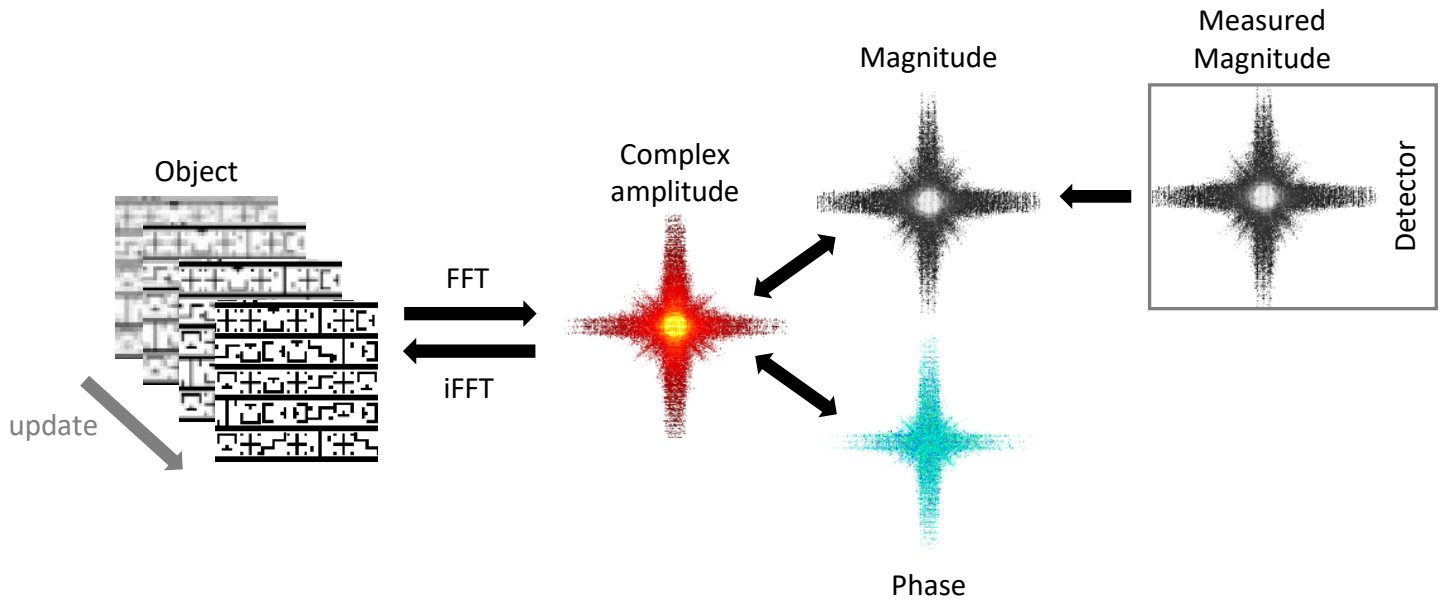

Figure 2. Working principle of iterative phase retrieval algorithms. More details on the reconstruction procedure can be found in the text.

As in ptychography many diffraction patterns are collected that correspond to the overlapping scan positions, the question remains how we treat all of the positions within the reconstruction procedure described above. Fig. 3 schematically illustrates the two different approaches how we can combine the information from all the positions. In the sequential approach, the object section illuminated by the first scan position is updated by replacing the estimated magnitude with the corresponding measured diffraction pattern magnitude (in the illustration 
turning from purple to yellow). When an adjacent scan position is considered, the object is already partially updated. Each object update benefits from a better initial guess which makes sequential algorithms generally faster (fewer iterations until convergence is reached) than parallel ones. ${ }^{8}$ Each position is updated one after the other until every scan position is updated once. Having updated each position once corresponds to one iteration. In the parallel update, all positions are considered and updated in parallel. Parallel solvers typically need more iterations to converge to a solution but are naturally structured for parallel execution on a suitable computer architecture, which makes them more efficient in terms of computation time, having a lower per-iteration time. ${ }^{8}$

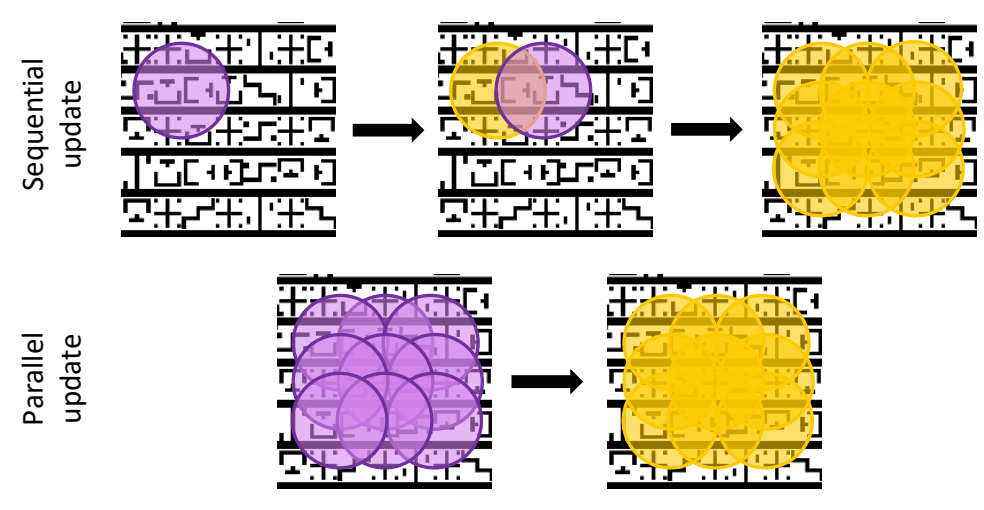

Figure 3. Illustration of the sequential versus parallel approach to update the object in a ptychography scan.

Today, the most common algorithms used for phase retrieval in ptychography are the difference map ${ }^{7}$ (DM) and the ptychographic iterative engine ${ }^{9}$ (PIE), where the latter describes a whole family of algorithms. The algorithms differ in the way they search for a solution to the phase problem, resulting in different behaviour, for example regarding noise, convergence efficiency, and requirements on the initial guess accuracy. To find the optimal strategy for RESCAN, we compare here the DM and the rPIE algorihtm to a recently reported method, the Least-squares maximum-likelihood (LSQML) approach. ${ }^{8}$

The DM algorithm is a parallel solver, known to be robust against systematic and random errors, such as image noise or probe positioning errors, that typically converges to a steady state without finding the optimal solution. ${ }^{7}$ For this study, we furthermore considered the rPIE algorithm, a member of the PIE family. ${ }^{9}$ It is a sequential solver designed to be more robust than the more common ePIE algorithm. The rPIE is still less robust against systematic and random errors than the DM method, ${ }^{8}$ nevertheless, when it converges, the rPIE algorithm is more likely to find the global optimum. ${ }^{8}$ The last algorithm for this comparison is the LSQML method that uses a block-update approach, which employs advantages of both, parallel and sequential solver. All scan positions are grouped into blocks of equal size, within one block, the positons are updated in parallel whereas one block is updated sequentially after the other. ${ }^{8}$ LSQML is the statistical optimal approach to the phase retrieval problem and reported to achieve the best imaging performance compared to the DM and PIE methods. ${ }^{8}$

\section{SIMULATION RESULTS}

For a first evaluation of the algorithm performance, we carried out a realistic simulation matching the characteristics and illumination conditions used in the RESCAN setup. We included a Poisson noise level consistent with the photon flux level used in RESCAN.

The object used in the simulations is a flat, binary mask obtained from the reticle design file of the sample described in Sec. 4. A region of about $40 \times 40 \mu \mathrm{m}^{2}$ with a pixel size of $34.44 \mathrm{~nm}$ is chosen for the simulation. The probe was generated as the image of the beam shaping aperture with a radius of $5 \mu \mathrm{m}$ on reticle and an illumination wavelength of $13.5 \mathrm{~nm}$ (EUV). To generate the diffraction patterns, the object was scanned by the probe in a circular scan pattern to avoid regular grid pathology ${ }^{7}$ with step size of $1 \mu \mathrm{m}$. 
Here, we consider four different reconstruction strategies. At first, we compare the performance of a parallel solver, the difference $\mathrm{map}^{7}(\mathrm{DM})$, to a sequential one, $\mathrm{rPIE},{ }^{9}$ running each for 100 iterations. Then, we compare again 100 iterations of DM to 100 iterations of rPIE each followed by 50 iterations of LSQML, ${ }^{8}$ to see whether a statistically optimal approach can further improve the solutions obtained with the DM or rPIE method.

To compare the algorithm performance, we considered two different error metrics. First, the Fourier error, which is commonly used (with various names) to report algorithm performance. ${ }^{9,10}$ The Fourier error is a measure of convergence:

$$
E_{\text {Fourier }}=\frac{\sum_{\mathbf{q}} \sum_{i, j}\left|\sqrt{I_{i j}}-\right| F\left[\Psi_{i j}\right]||}{\sum_{\mathbf{q}} \sum_{i, j} 1},
$$

where $I$ is the intensity measured on the detector and $\Psi$ is the current exit wave guess. $F$ corresponds to the Fourier transform operator which, in this case, approximates well the propagation of the light in the far-field. ${ }^{11}$ In the iterative reconstruction procedure described in more detail in Sec. 2, the magnitude of the complex amplitude (the Fourier transform of the exit wave) is replaced by the square root of the measured intensity on the detector. Thus, as the difference between the estimate and the measured amplitude gets smaller, the Fourier error decreases and the algorithm approaches a solution. The Fourier error is summed over all pixels $(i, j)$ and averaged over the scan positions $(\mathbf{q})$. Here, only the inner scan positions are considered for averaging to avoid artifacts from the boundary regions of the scan.
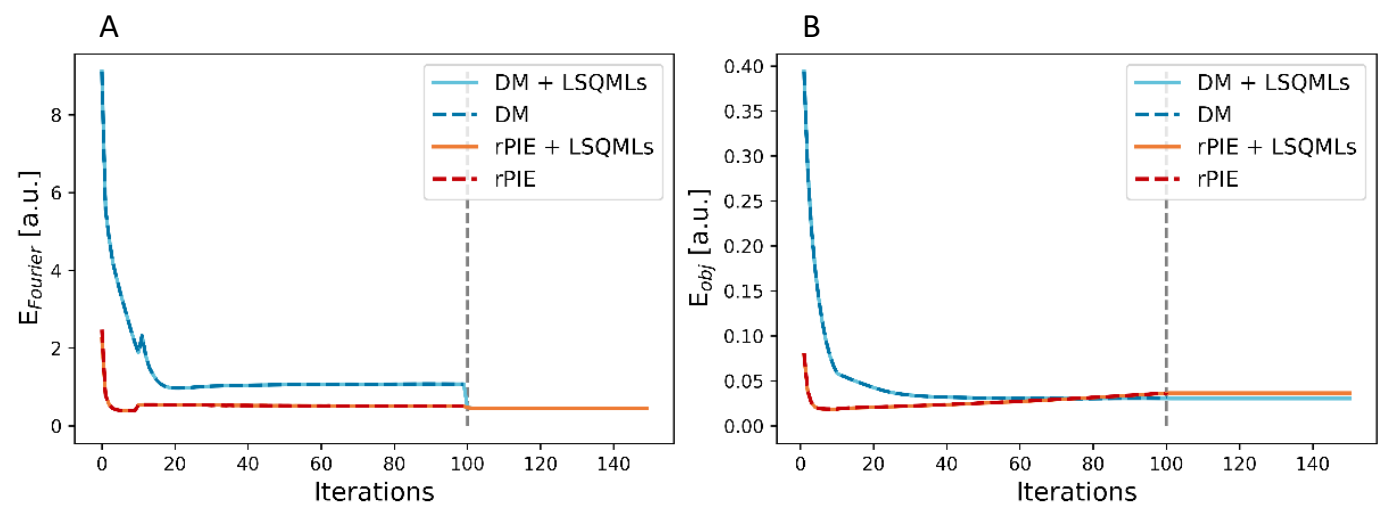

Figure 4. A Fourier error for the reconstruction cases considered in the simulation: DM, DM + LSQMLs, rPIE and rPIE + LSQMLs. The s in LSQMLs stands for a sparse grouping in the block-update approach for the LSQML algorithm. ${ }^{8} \mathbf{B}$ Object error for the same cases as shown in A.

In Fig. 4A the Fourier error for all four cases is decreasing until it stagnates at a constant error. It is important to note that the curves for the DM and DM+LSQML reconstructions lie exactly on top of each other, as the ones for rPIE and rPIE+LSQML. Convergence for all four cases is reached after about 20 iterations with the error for both rPIE runs stagnating even a bit earlier. As described in Sec. 2 this is a typical characteristic of sequential algorithms compared to parallel ones. In both cases, for rPIE+LSQML and DM+LSQML, the Fourier error is decreasing further as soon as the LSQML algorithm is started after the first 100 iterations (dashed line in Fig. 4A).

From the Fourier error curves one would consider the rPIE algorithm as optimal choice for the object reconstruction as it converges in the fewest iterations and additional runs with LSQML seem only to bring a minor improvement in the error. But as we are more concerned about the image quality than the convergence behaviour for EUV mask metrology, we need to consider a second error metric, here named the object error $E_{\text {obj }}$

$$
E_{\text {obj }}=\frac{\sum_{\mathbf{r}} \sum_{i, j}|| O_{i j}^{r e f}|-| \hat{O}_{i j} \|^{2}}{\sum_{\mathbf{r}} \sum_{i, j} 1},
$$


which compares the reconstructed object $\left(\hat{O}_{i j}\right)$ to a reference image $\left(O_{i j}^{r e f}\right)$ which is obtained from the reticle design. This metric is similar to the simulation error $E_{\text {sim }}$ introduced by Maiden et al. ${ }^{12}$ For the simulation described here, the object from which the diffraction patterns were generated is used as reference.

The object error for all four simulation cases is shown in Fig. 4B. Similarly to the Fourier error, the DM and rPIE algorithm reach a constant error level after the first 20 iterations. Surprisingly, the reconstructed image quality for the rPIE algorithm is lowest after ca. 5 iterations and slightly decreases (larger error) for further iterations. The most important difference to the Fourier error is observed as soon as the LSQML algorithm is started (dashed grey line) after the first 100 iterations of DM or rPIE. The error is not decreasing further but remains on the same level as reached from the previous DM and rPIE iterations. We therefore conclude that for the simulations performed here, the LSQML algorithm is not further optimizing the image quality and does not lead to a lower object error.

\section{EXPERIMENTAL RESULTS}

To compare our findings of the previous section to experimental data, we measured an EUV mask manufactured ${ }^{4}$ using the same design of the object used in the simulation. The scan pattern and step size, as well as other reconstruction parameters are the same as in the simulation described above. The object error for a reconstruction using first 300 iterations of DM respectively rPIE, with additional 100 iterations of LSQML is shown in Fig. 5. The reference object is generated with the mask design file ${ }^{13}$ and is aligned and scaled to match the inspected area. ${ }^{4}$

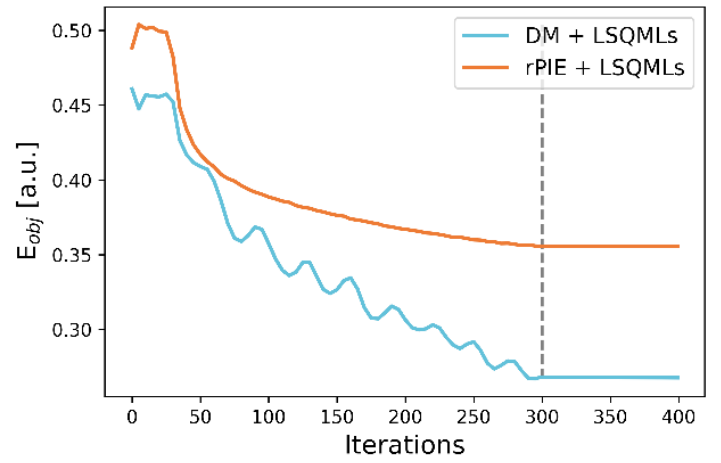

Figure 5. Object error for each 300 iterations of DM or rPIE with additional 100 iterations of LSQMLs. The data was collected using the corresponding EUV reticle to the mask design file used in the simulation.

The object error is decreasing for both cases within the first 300 iterations without stagnating to a fixed error level. This implies that additional iterations with DM and rPIE could have improved the image quality further. In contrast to the simulation case, the DM algorithm is performing better for the experimental data than rPIE. The DM is reconstructing in fewer iterations to a lower object error. For both cases, as soon as the LSQML iterations are started (after the first 300 iterations, indicated by the dashed grey line), the object error is not decreasing but remains at the same level as the last rPIE resp. DM iteration.

In Fig. 6, the reconstructed object magnitude is shown after 300 iterations of DM (A) and additional 100 iterations of LSQML (B) as well as for 300 iterations of rPIE (C) and also additional 100 iterations LSQML (D). The difference map algorithm reconstructs the main features of the reticle, the power lines and the structures in between are clearly visible. The reconstruction quality is not optimal as some phase artifacts cause vertical stripes in the magnitude of the reconstructed object. Further DM iterations could improve this reconstruction, as well as additional modifications of the algorithm to account for illumination wavefront instabilities or sample drift. ${ }^{14-17}$ From Fig. $6 \mathrm{~B}$ one can see that rPIE failed to reconstruct the object. Even though one can guess some of the structure shapes as the crosses or the power lines, the reconstructed image quality is unacceptable 
for mask metrology. As the corresponding object error (see Fig. 5) is converging, the algorithm seems to get stuck in a local minimum not finding the optimal solution. As rPIE is known to be less robust to systematic and random errors than the DM algorithm ${ }^{8}$, we believe that the error level in the experimental data case was too high for a high quality reconstruction using rPIE. With an improved initial guess that is starting closer to the optimal solution, rPIE could perform better than here reported. This remains an open question for further investigation.

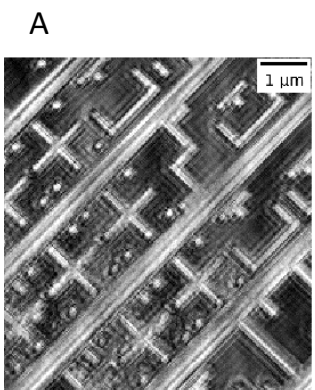

DM
B

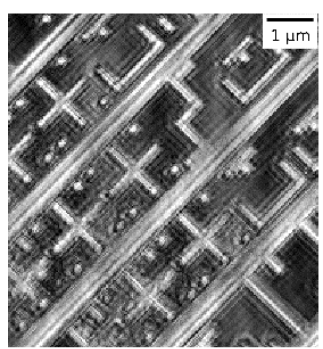

$\mathrm{DM}+\mathrm{LSQML}$

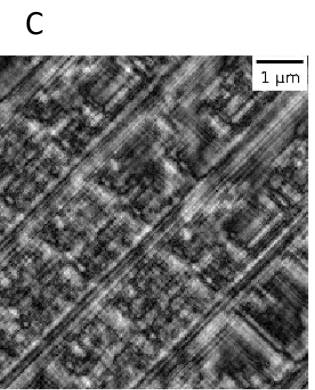

rPIE

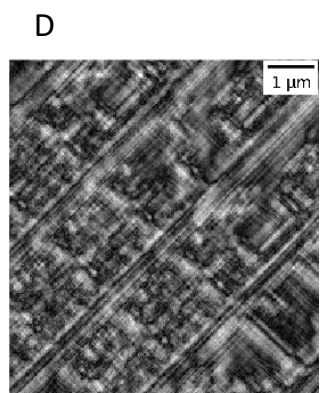

rPIE + LSQML

Figure 6. Reconstructed image magnitude after 300 iterations of DM (A) and additional 100 iterations of LSQML (B) and 300 iterations of rPIE (C) with additional 100 iterations of LSQML (D).

\section{CONCLUSIONS AND OUTLOOK}

In this study, we compared different algorithms to identify the optimal image reconstruction strategy for the RESCAN microscope. First, we compared the performance of the parallel $\mathrm{DM}^{7}$ algorithm to the sequential $\mathrm{rPIE}^{9}$ solver. Additionally, we studied if following iterations using the statistically optimal LSQML ${ }^{8}$ approach result in further image quality improvement.

We observed that the Fourier error, which measures the converge of the algorithm, is not suited for a comparison of the reconstructed image quality. Therefore, we introduced a second error metric, the object error $E_{\mathrm{obj}}$, that measures the reconstructed image quality with respect to a reference. From both the simulations and the experimental data reconstructions, we couldn't observe a significant improvement in reconstructed object quality with additional iterations using the LSQML algorithm. In the experimental data case, only the DM algorithm reconstructed the reticle image, whereas the rPIE algorithm failed to reconstruct the experimental data with the required accuracy. Therefore, our current best strategy is to use the DM as standard reconstruction approach. The difference map algorithm has the additional advantage that it can be optimized for per iteration speed, as it is parallelizable on a suitable computational architecture.

We will continue to investigate different reconstruction strategies for the RESCAN microscope to find the optimal approach for higher image reconstruction quality and reconstruction speed.

\section{ACKNOWLEDGMENTS}

The authors would like to thank Markus Kropf, Michaela Vockenhuber and José Gabadinho for their technical support at the XIL-II beamline at the SLS.

\section{REFERENCES}

[1] Mochi, I., Helfenstein, P., Mohacsi, I., Rajeev, R., Kazazis, D., Yoshitake, S., and Ekinci, Y., "RESCAN: an actinic lensless microscope for defect inspection of EUV reticles," J. Micro/Nanolithography, MEMS, MOEMS 16(4), 041003 (2017).

[2] Chapman, H. N. and Nugent, K. A., "Coherent lensless X-ray imaging," Nat. Photonics 4(12), 833-839 (2010). 
[3] Helfenstein, P., Mochi, I., Rajeev, R., Fernandez, S., and Ekinci, Y., "Coherent diffractive imaging methods for semiconductor manufacturing," Adv. Opt. Technol. 6(6), 439-448 (2017).

[4] Mochi, I., Fernandez, S., Nebling, R., Locans, U., Helfenstein, P., Rajeev, R., Dejkameh, A., Kazazis, D., Tseng, L.-T., and Ekinci, Y., "Amplitude and phase defect inspection on EUV reticles using RESCAN," Extrem. Ultrav. Lithogr. X (March), 29 (2019).

[5] Hegerl, R. and Hoppe, W., "Phase evaluation in generalized diffraction (ptychography)," in [Proc. Fifth Eur. Congr. Electron Microsc.], 628 - 629 (1972).

[6] Rodenburg, J. M., "Ptychography and related diffractive imaging methods," Adv. Imaging Electron Phys. 150(07), 87-184 (2008).

[7] Thibault, P., Dierolf, M., Bunk, O., Menzel, A., and Pfeiffer, F., "Probe retrieval in ptychographic coherent diffractive imaging," Ultramicroscopy 109(4), 338-343 (2009).

[8] Odstrčil, M., Menzel, A., and Guizar-Sicairos, M., "Iterative least-squares solver for generalized maximumlikelihood ptychography," Opt. Express 26(3), 3108-3123 (2018).

[9] Maiden, A., Johnson, D., and Li, P., "Further improvements to the ptychographical iterative engine," Optica 4(7), 736 - 745 (2017).

[10] Maiden, A. M. and Rodenburg, J. M., "An improved ptychographical phase retrieval algorithm for diffractive imaging," Ultramicroscopy 109(10), 1256-1262 (2009).

[11] Goodman, J. W., [Introduction to Fourier Optics], vol. 35, Roberts \&Company, Englewood, Colorado, 3 ed. (1996).

[12] Maiden, A., Johnson, D., and Li, P., "Further improvements to the ptychographical iterative engine SOM1," Optica 4(7), 736 (2017).

[13] Mochi, I., Helfenstein, P., Rajeev, R., Fernandez, S., Kazazis, D., Yoshitake, S., and Ekinci, Y., "Actinic inspection of EUV reticles with arbitrary pattern design," Proc. SPIE - Int. Soc. Opt. Eng. 10450(October 2017) (2017).

[14] Whitehead, L. W., Williams, G. J., Quiney, H. M., Vine, D. J., Dilanian, R. A., Flewett, S., Nugent, K. A., Peele, A. G., Balaur, E., and McNulty, I., "Diffractive imaging using partially coherent X rays," Phys. Rev. Lett. 103(24), 243902 (2009).

[15] Thibault, P. and Menzel, A., "Reconstructing state mixtures from diffraction measurements," $N a$ ture 494(7435), 68-71 (2013).

[16] Odstrcil, M., Baksh, P., Boden, S. A., Card, R., Chad, J. E., Frey, J. G., and Brocklesby, W. S., "Ptychographic coherent diffractive imaging with orthogonal probe relaxation," Opt. Express 24(8), 8360-8369 (2016).

[17] Helfenstein, P., Rajeev, R., Mochi, I., Kleibert, A., Vaz, C. A. F., and Ekinci, Y., "Beam drift and partial probe coherence effects in EUV reflective-mode coherent diffractive imaging," Opt. Express 26(9), 1224212256 (2018). 